\title{
Comparison between two approaches to modeling microsatellite DNA repeats: infinite dimensional model and its $n$-dimensional approximation
}

\author{
MAŁGORZATA BIAŁKA
}

\begin{abstract}
Two approaches to modeling microsatellite DNA repeats are considered. The former is an infinite dimensional system based on the theory of branching random walks which dynamic properties are characterized using Laplace transforms and Laplace asymptotic techniques. The latter is an $n$-dimensional approximation where microsatellite DNA repeats model is the example of a chain system. Both models were the subject of many numerical calculations using the MATLAB software. The results allow us to evaluate the asymptotic behavior and determine the effect of the system parameters on the run of the solution and the state variables.
\end{abstract}

Key words: infinite-dimensional systems, asymptotic stability, chain systems, microsatellites DNA repeats, polymerase slippage

\section{Introduction}

Microsatellites are the short tandem repeats of motifs of length up to 6 pairs of bases. Their high polymorphism is shown in the variable structure of basic sequence and in the length of repeated sequence. Microsatellites are widely dispersed throughout the eukaryotic genome. The lengths of STR sequences appear to be stable during the life of an individual. Changes in microsatellite sequences are not the result of classic point mutations where a single nucleotide is exchanged by another. Alteration in the length of microsatellite sequences takes place in the process called polymerase slippage resulting in lengthening or shortening the sequence by one motif depending on which polymerase strand an error occurred [1].

Although the function of short tandem repeats has not been defined yet, they are very useful for various genetics applications. Microsatellites are important tool in forensics (genetic fingerprinting of individuals), paternal analysis, molecular phylogenetics, etc.. They play a significant role in early diagnosis of some cancers because their length changes in the early disease development. This phenomenon is known as microsatellite instability (MI) . In oncology the meaning of MI was described for the first time

The author is with the Institute of Automatic Control, Silesian University of Technology, Gliwice, Poland. E-mail: mbialka1240@wp.pl

Received 28.10.2011. Revised 17.12.2011. 
in hereditary nonpolyposis colon cancer. Microsatellite instability is present in 55-86\% cases of HNCC. Frequency of MI in sporadic colon cancers is significant either - it varies from 15 to $35 \%$ [2]. The tri-nucleotides microsatellites repeats which length exceeds a normal range are responsible for various genetic disorders: Huntington disease, fragile X, myotonic dystrophy, spinal-bulbar muscular atrophy or Friedreich's ataxia. Huntington disease is the example of polyglutamine expansions. Human gene HD coding huntingtin protein contains the sequence CAG repeated from 10 to 35 times. In the case of Huntington disease the number of repeats increases from 36 to 121 resulting in protein malfunction and disease development [1].

The evolution of microsatellite DNA repeats can be described using an infinite system of linear differential equations [3]. The use of Laplace transforms and asymptotic Laplace expansions allow us to analyze the stability of the model. The n-dimensional approximation is an example of chain system with tridiagonal system matrix [4]. The present paper shows the results of numerical studies using the MATLAB software for both approaches to modeling microsatellites mentioned above. In the literature we can find another techniques applied to modeling microsatellites (e.g. [5], [6]). In paper [5] the considered model combines stepwise changes of the length of STR sequence with the possibility of sequence breakdown and the problem is solved using the theory of Markov chains and stochastic semigroups.

\section{Modeling microsatellite DNA repeats}

We consider microsatellites as a stratified population of individuals which differ in number of repeats of basic sequence built up to 6 pairs of bases. Let us index the subpopulations $i=0,1,2, \ldots$.The time evolution of population can be described by a branching random walk with an absorbing boundary as it follows [3]:

- the quantity of types of particles is unlimited: $i=0,1,2, \ldots$,

- particle's lifetime is described by $\theta$ which is the parameter of exponential distribution,

- each particle in the moment of its death produces the pair of progeny; the single progeny particle survives independently with probability $\beta$,

- the progeny of 0 type particle is also of type 0 ,

- the progeny of $i$ type particle, $i=1,2, \ldots$ became of type $i-1$ with probability $v$, type $i+1$ with probability $\eta$ and type $i$ with probability $1-v-\eta$.

We assume that at the time $t=0$ there exists $N_{i}(0) i$ type particles. We denote $N_{i}(t)$ the quantity of $i$ type particles at the time $t$. We also denote:

$$
d=2 \beta \nu \theta, \quad b=2 \beta \eta \theta, \quad \lambda=(2 \beta-1) \theta .
$$


We obtain the following infinite system of linear differential equations:

$$
\left\{\begin{array}{l}
\dot{N}_{0}(t)=\lambda N_{0}(t)+d N_{1}(t) \\
\dot{N}_{1}(t)=\lambda N_{1}(t)-(b+d) N_{1}(t)+d N_{2}(t) \\
\vdots \\
\dot{N}_{i}(t)=\lambda N_{i}(t)-(b+d) N_{i}(t)+d N_{i+1}(t)+b N_{i-1}(t) \quad i \geqslant 2 \\
\vdots
\end{array}\right.
$$

We assume that:

$$
d>0, \quad b>0, \lambda>0, \text { and } d>b .
$$

Only the first equation contains element $N_{0}(t)$ so we can limit our considerations to the system in the form:

$$
\left\{\begin{array}{l}
\dot{N}_{1}(t)=\lambda N_{1}(t)-(b+d) N_{1}(t)+d N_{2}(t) \\
\vdots \\
\dot{N}_{i}(t)=\lambda N_{i}(t)-(b+d) N_{i}(t)+d N_{i+1}(t)+b N_{i-1}(t) \quad i \geqslant 2 \\
\vdots
\end{array}\right.
$$

\section{Asymptotic behavior of infinite dimensional model}

The solution of the infinite dimensional system (4) was obtained using Laplace transforms under following assumptions: $d \neq b$ and the initial conditions: $N_{i}(0)=\delta_{i j}$, where $\delta$ is Kronecker delta. The final result has the following form [3]:

$$
\begin{gathered}
N_{1}(t)=\left(\frac{k(\sqrt{d / b})^{k}}{d}\right) \frac{I_{k}(2 \sqrt{b d} t)}{t} e^{[\lambda-(b+d)] t} \\
N_{\Sigma}(t)=e^{\lambda t}-e^{\lambda t} k(\sqrt{d / b})^{k} \int_{0}^{t} \frac{I_{k}(2 \sqrt{b d} \tau)}{\tau} e^{-(b+d) \tau} d \tau
\end{gathered}
$$

To examine the behavior of the solution in long time horizon we consider the asymptotic solution of the infinite dimensional system (4) which was obtained using Laplace asymptotic expansions $[3,7,8]$. Finally we obtain:

$$
N_{1}(t) \sim \frac{1}{2 \sqrt{\pi} \sqrt[4]{(b d)^{3}}} t^{-3 / 2} e^{\left[\lambda-(\sqrt{d}-\sqrt{b})^{2}\right] t}
$$




$$
N_{\Sigma}(t) \sim\left[1-\frac{\min (b, d)}{b}\right] e^{\lambda t}+\frac{d}{2 \sqrt{\pi} \sqrt[4]{(b d)^{3}}(\sqrt{d}-\sqrt{b})^{2}} t^{-3 / 2} e^{\left[\lambda-(\sqrt{d}-\sqrt{b})^{2}\right] t} .
$$

Having the formulas (7) and (8) we can easily precise the conditions of convergence of the system (4) to zero. The solution of the system (4) decays exponentially to 0 , as $t \rightarrow \infty$ if and only if:

$$
d>b, \text { and } \sqrt{d}-\sqrt{b}>\sqrt{\lambda} .
$$

To simulate the approximate solution we take long time horizon which assures results corresponding to the behavior of the accurate solution. The parameter values have been taken from [9].

Figures 1 and 2 show the trajectory of the approximate output $N_{\Sigma}(t)$ and the approximate state variable $N_{1}(t)$ under conditions (9). Figures 3 and 4 show the case where convergence conditions are not satisfied and the number of particles grows quickly to infinity. Figures 5 and 6 concerns the case where condition $\sqrt{d}-\sqrt{b}>\sqrt{\lambda}$ is not satisfied and $d>b$ so the growth is slower.

The numerical studies of the accurate solution enable us to determine the influence of parameters. Figures 7 and 8 show the relationship between the trajectory of the solution $N_{\Sigma}(t)$ and $N_{1}(t)$ for different values of parameter $d$ which depends on probability $v$ that the progeny of $i$ particle will be type $i-1$. Parameter values which have bee taken for investigations: $k=1, b=0.2, \lambda=0.1, d=0.5,0.9,1.1$ and 1.7.

We can observe that if $d$ is smaller then the solution is closer to instability region.

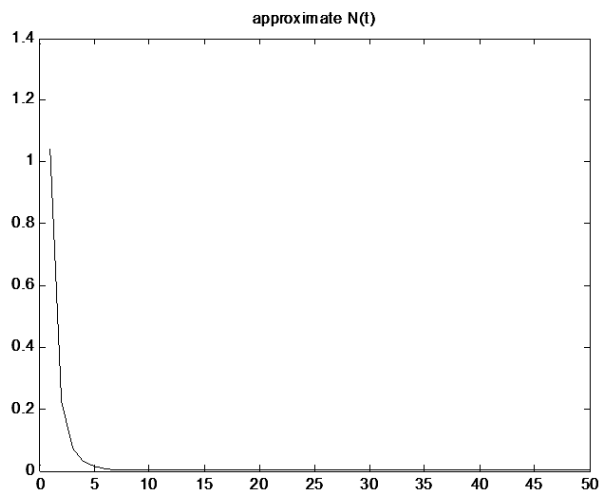

Figure 1. The approximate output $N_{\Sigma}(t)$ for $b=0.2, d=1.5$ and $\lambda=0.1$. 


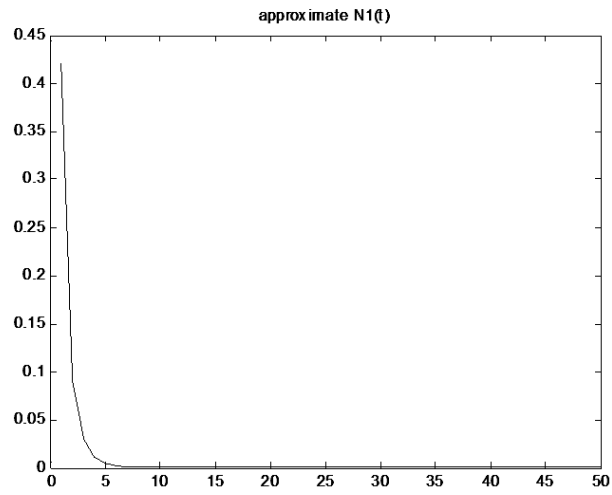

Figure 2. The approximate state variable $N_{1}(t)$ for $b=0.2, d=1.5$ and $\lambda=0.1$.

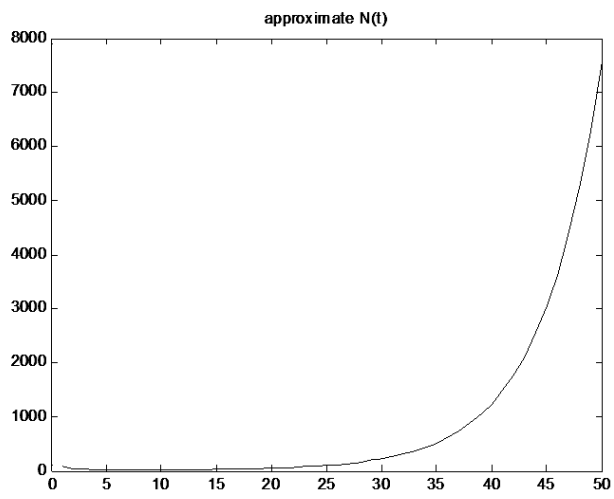

Figure 3. The approximate output $N_{\Sigma}(t)$ for $b=0.5, d=0.4$ and $\lambda=0.2$.

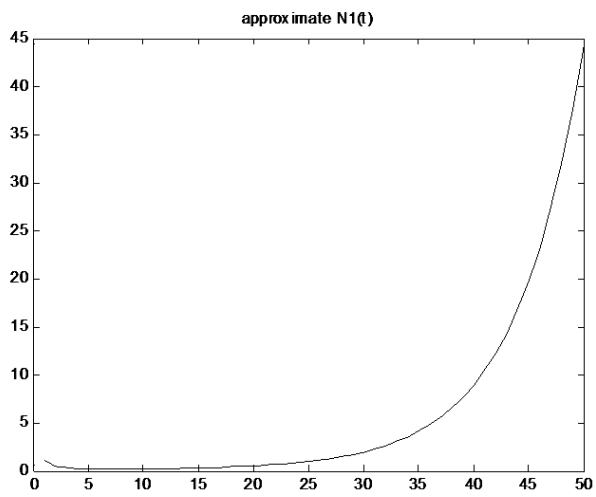

Figure 4. The approximate state variable $N_{1}(t)$ for $b=0.5, d=0.4$ and $\lambda=0.2$. 


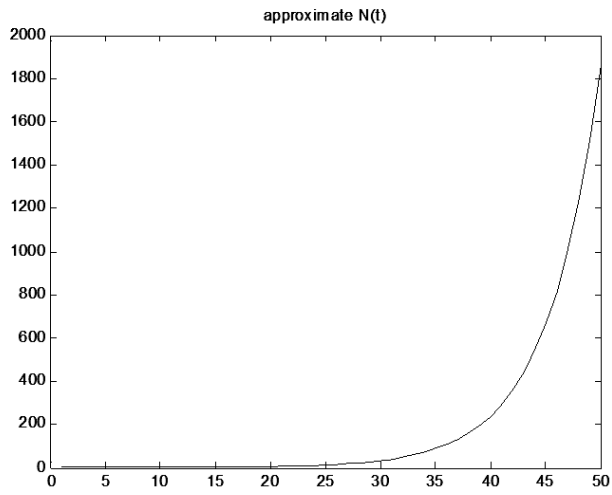

Figure 5. The approximate output $N_{\Sigma}(t)$ for $b=0.3, d=0.9$ and $\lambda=0.4$.

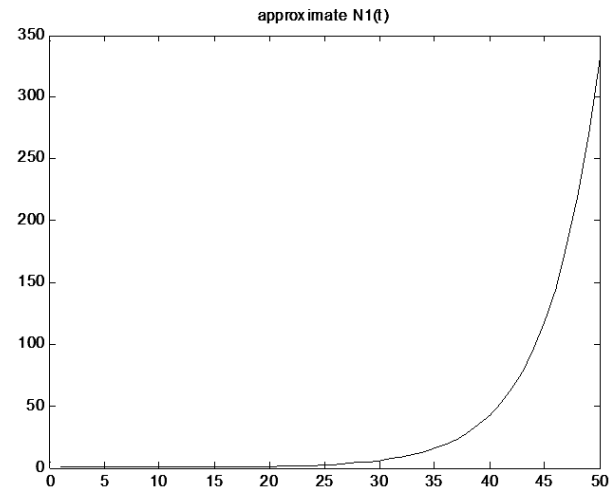

Figure 6. The approximate state variable $N_{1}(t)$ for $b=0.3, d=0.9$ and $\lambda=0.4$.

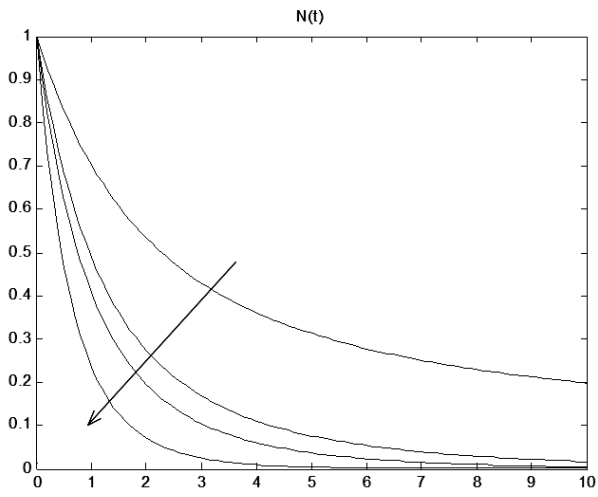

Figure 7. $N_{\Sigma}(t)$ - influence of parameter $d$. 


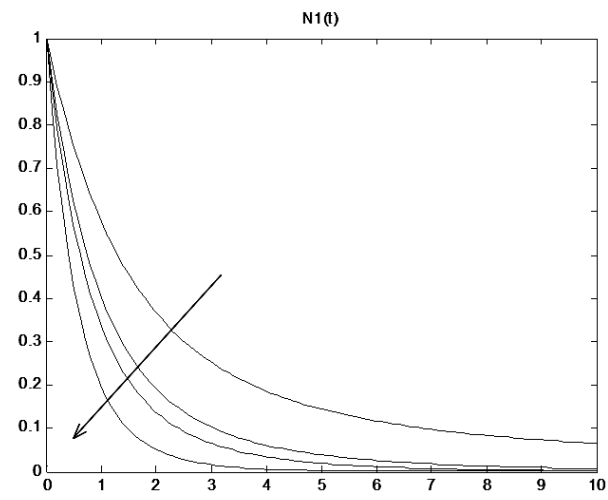

Figure 8. $N_{1}(t)$ - influence of parameter $d$.

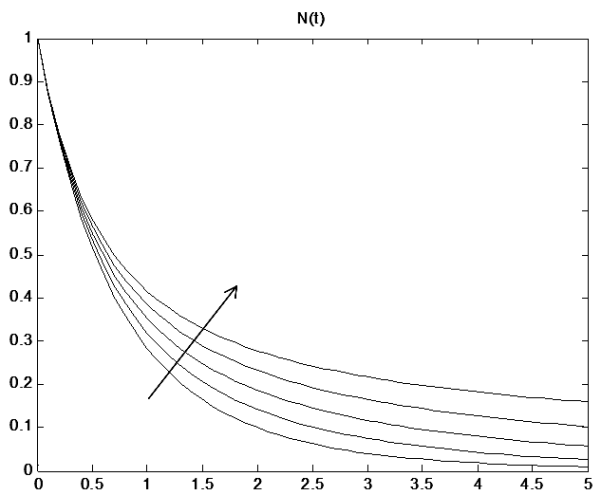

Figure 9. $N_{\Sigma}(t)$ - influence of parameter $b$.

Figures 9 and 10 show the influence of different values of $b$ on the solution. Parameter $b$ depends on probability $\eta$ with which the progeny of $i$ type particle become of $i+1$ type. The increase of $b$ causes instability. Parameter values which have bee taken for investigations: $k=1, d=1.5, \lambda=0.1, b=0.2,0.4,0.6,0.8,1$.

Now we examine the influence of parameter $\lambda$ depending on probability of progeny surviving $\beta$ and coefficient of exponential distribution of particles life-time. The results are shown in Figs 11 and 12. Parameter values which have bee taken for investigations: $k=1, b=0.2, d=1.5, \lambda=0.1,0.3,0.5,0.6,0.7$. The growth of $\lambda$ leads to instability.

Let us check how the trajectory of the output changes for different index $k$ of nonzero initial condition. This relationship is presented in Figs 13 and 14 for $k=1,2,3,4,5$ and in Figs 15 and 16 for $k=6,7,8,9,10$. Parameter values are: $b=0.2, d=1.5, \lambda=0.1$. The increase of $k$ implies the later decay of $N_{\Sigma}(t)$ and $N_{1}(t)$. 


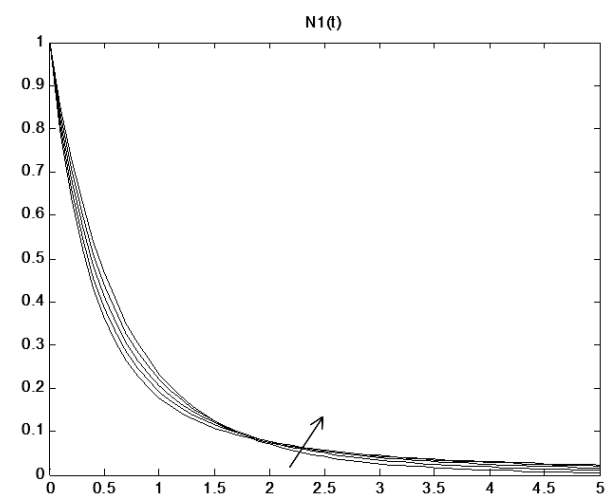

Figure 10. $N_{1}(t)$ - influence of parameter $b$.

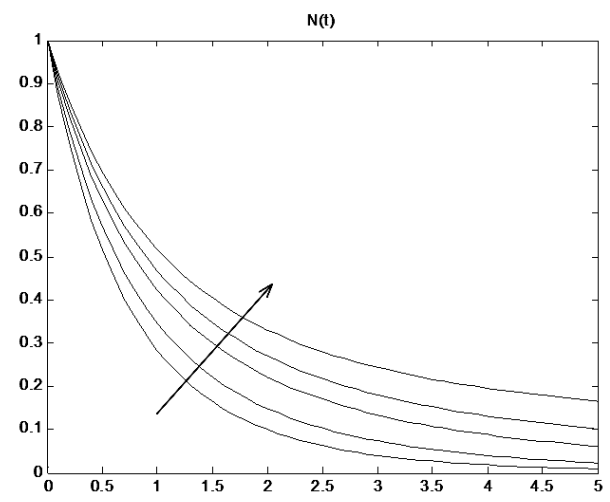

Figure 11. $N_{\Sigma}(t)$ - influence of parameter $\lambda$.

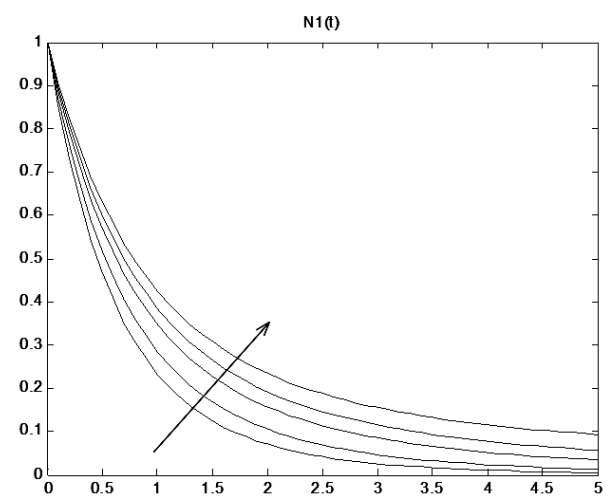

Figure 12. $N_{1}(t)$ - influence of parameter $\lambda$. 


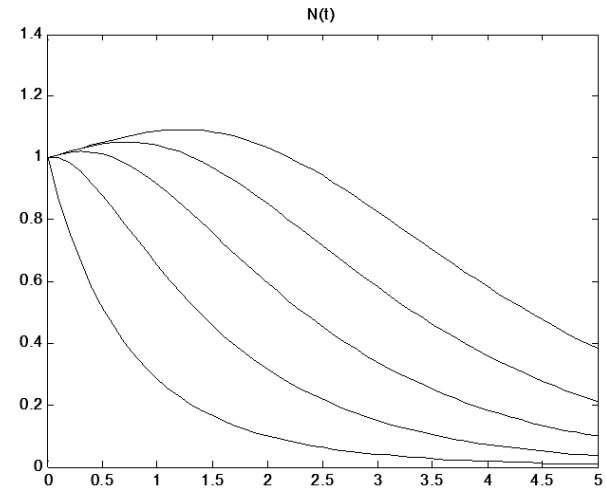

Figure 13. $N_{\Sigma}(t)$ - influence of parameter $k$.

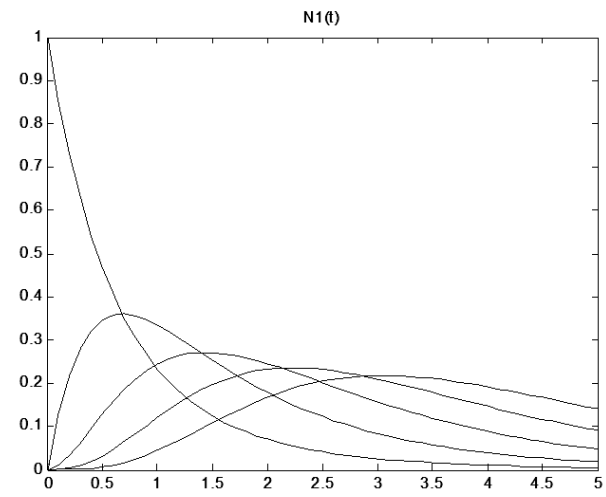

Figure 14. $N_{1}(t)$ - influence of parameter $k$.

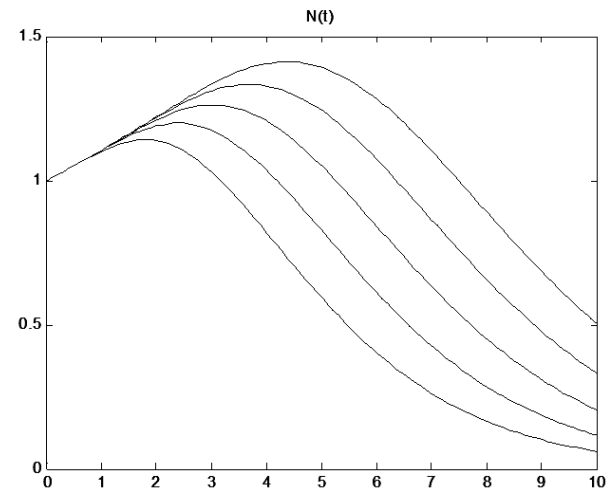

Figure 15. $N_{\Sigma}(t)$ - influence of parameter $k$. 


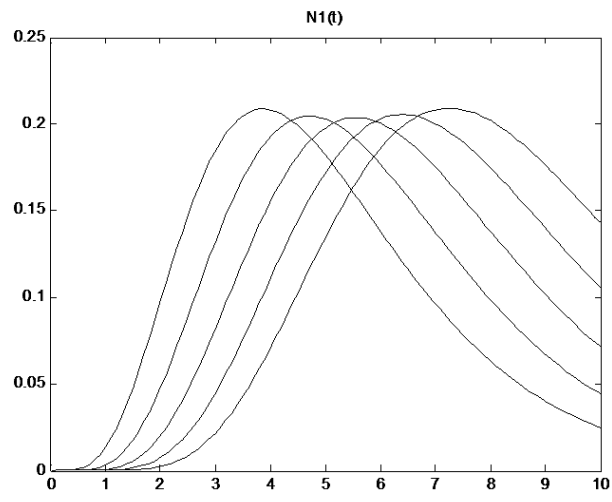

Figure 16. $N_{1}(t)$ - influence of parameter $k$.

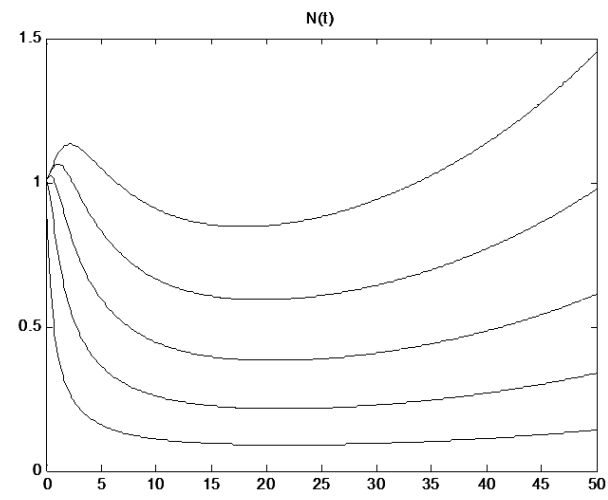

Figure 17. The output $N_{\Sigma}(t)$ for $k=1,2,3,4,5$.

Figures 17 and 18 present the trajectory of $N_{\Sigma}(t)$ and $N_{1}(t)$ for $k=1,2,3,4,5$ when $d>b$ but the second condition $\sqrt{d}-\sigma b>\sqrt{\lambda}$ is not satisfied $(d=1.5, b=1, \lambda=0.1)$.

Figures 19 and 20 show the situation when $d<b$ for $k=1,2,3,4,5(d=0.8, b=1$, $\lambda=0.1)$. We can observe that the output $N_{\Sigma}(t)$ grows immediately and does not lose the value in the beginning of the trajectory.

Finally, the main observation is that even in the case of unstable trajectories of $N_{\Sigma}(t)$ and $N_{1}(t)$ in a short time horizon we can see decrease of the number of particles. The growth takes place later. Therefore there is necessity to check the behavior of the examined quantities for the long time horizon. 


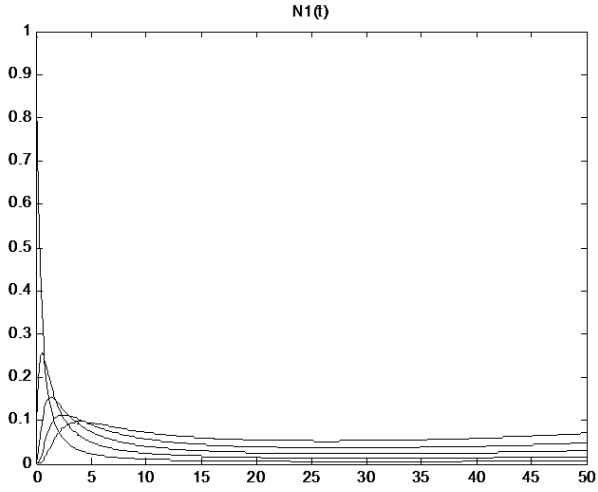

Figure 18. The state variable $N_{1}(t)$ for $k=1,2,3,4,5$.

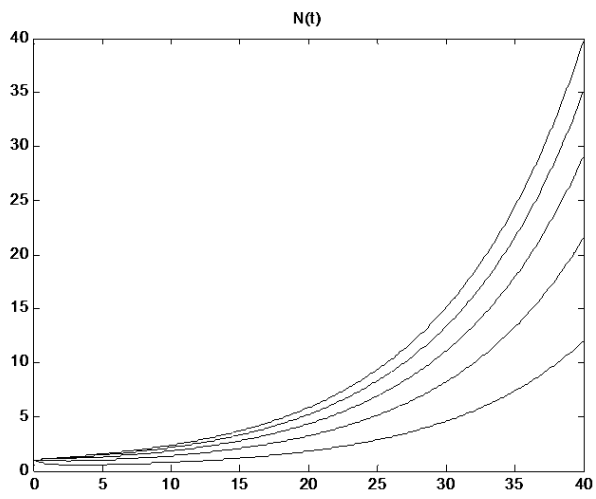

Figure 19. The output $N_{\Sigma}(t)$ for $k=1,2,3,4,5$.

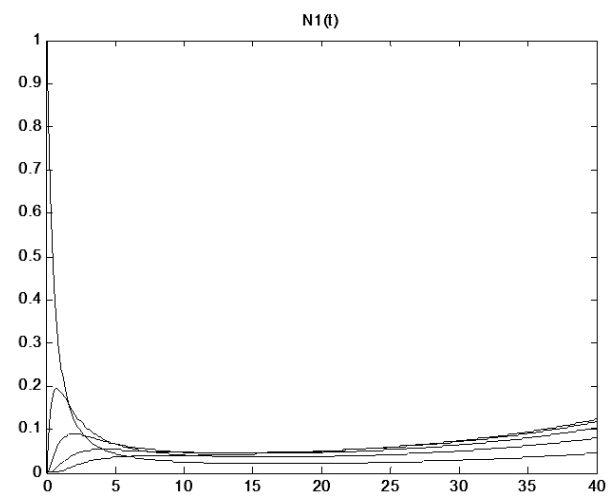

Figure 20. The state variable $N_{1}(t)$ for $k=1,2,3,4,5$. 


\section{4. n-dimensional approximation}

The infinite dimensional system (4) reduced to $n$-dimensional model has the following form:

$$
\left\{\begin{array}{l}
\dot{N}_{0}(t)=\lambda N_{0}(t)+d N_{1}(t) \\
\dot{N}_{1}(t)=\lambda N_{1}(t)-(b+d) N_{1}(t)+d N_{2}(t) \\
\vdots \\
\dot{N}_{i}(t)=\lambda N_{i}(t)-(b+d) N_{i}(t)+d N_{i+1}(t)+b N_{i-1}(t) \quad i=2, \ldots, n-1 \\
\vdots \\
\dot{N}_{n}(t)=\lambda N_{n}(t)-(b+d) N_{n}(t)+b N_{n-1}(t)
\end{array}\right.
$$

We can write it in the form [4]:

$$
\begin{aligned}
& \dot{N}_{0}(t)=\lambda N_{0}(t)+d N_{1}(t) \\
& \dot{N}(t)=\left[\begin{array}{lll}
\lambda I+A_{n}
\end{array}\right] N(t) \\
& N(t)=\left[\begin{array}{lll}
N_{1}(t) & N_{2}(t) \ldots N_{n}(t)
\end{array}\right]^{T}
\end{aligned}
$$

where $A_{n}$, for $n=5$ takes the following form:

$$
A_{5}=\left[\begin{array}{ccccc}
-(b+d) & d & 0 & 0 & 0 \\
b & -(b+d) & d & 0 & 0 \\
0 & b & -(b+d) & d & 0 \\
0 & 0 & b & -(b+d) & d \\
0 & 0 & 0 & b & -(b+d)
\end{array}\right]
$$

We assume known initial condition vector: $N_{i}(0) \geqslant 0$. The system (11) serves as an example of the chain system. In this case we have the following analytic formula [10] for eigenvalues of $\left[\lambda I+A_{n}\right]$ :

$$
\lambda_{k}=\lambda-(b+d)+2 \sqrt{b d} \cos \varphi_{k}, \quad \varphi_{k}=\frac{k \pi}{n+1}, \quad k=1,2 \ldots, n .
$$

Consequently, we have:

$$
\lambda_{1}=\lambda-(\sqrt{b}-\sqrt{d})^{2}-2 \sqrt{b d}\left(1-\cos \frac{\pi}{n+1}\right)<1
$$

and

$$
\lambda_{n}<\lambda_{n-1}<\cdots<\lambda_{1}<\lambda
$$


The eigenvector of $\left[\lambda I+A_{n}\right]$ is given by $[10]$ :

$$
\begin{gathered}
w_{k}=\left[\begin{array}{lll}
w_{k, 1} & w_{k, 2} \ldots w_{k, n}
\end{array}\right]^{T} \\
w_{k, i+1}=\sqrt{\left(\frac{b}{d}\right)^{i}} \sin \frac{(i+1) k \pi}{n+1}, \quad i=0,1, \ldots, n-1, \quad k=1,2, \ldots, n .
\end{gathered}
$$

General solution of considered system (11) has the following form:

$$
\begin{gathered}
N_{0}(t)=e^{\lambda t} N_{0}(0)+\int_{0}^{t} e^{\lambda(t-\tau)} d N_{1}(\tau) d \tau \\
N(t)=e^{\left[\lambda I+A_{n}\right] t} N(0) .
\end{gathered}
$$

Therefore we obtain the following asymptotic stability conditions of the system (11):

$$
0 \leqslant \beta<0.5 \text {. }
$$

The condition (18) means the extinction of the whole considered population.

The system describing population of microsatellite DNA repeats

$$
\dot{N}(t)=\left[\lambda I+A_{n}\right] N(t)
$$

where $N(t)=\left[N_{1}(t) N_{2}(t) \ldots N_{n}(t)\right]^{T}$ is asymptotically stable if and only if:

$$
\lambda_{k}<0, \quad k=1,2, \ldots n .
$$

Finally, we obtain (see (12) and (14)) [4]:

$$
\beta<\beta_{1}=\frac{0.5}{1-\eta-v+2 \sqrt{\eta v} \cos \frac{\pi}{n+1}} .
$$

If $\beta<\beta_{1 \infty}=\frac{0.5}{1-\eta-v+2 \sqrt{\eta v}}$ the system is asymptotically stable for any $n$.

Figures 21, 22 and 23 present the behavior of the population $N_{0}$ for $\beta=0.45,0.5$ and 0.55 respectively. Values of the other parameters are: $n=5, v=0.45, \eta=0.01, \theta=0.9$, vector of initial conditions $N(0)=\left[\begin{array}{lllll}0.1 & 0.1 & 0.1 & 0.1 & 0.1\end{array}\right], N_{0}(0)=0$.

Figures 24, 26 and 28 present influence of different values of $\beta$ on behavior of the sum of particles type $i=0,1, \ldots, 5$ and each type separately. Values of other parameters are the same as above.

In Fig. 26, for $\beta=0.5$ the trajectory of the sum of particles settles on certain value, while the particles of type $i=1,2, \ldots, 5$ (Fig. 27) decay. For $\beta=0.55$ the sum in Fig. 28 loses stability because it contains particles of 0 type, while the trajectories of types $1,2, \ldots, 5$ remain stable. 


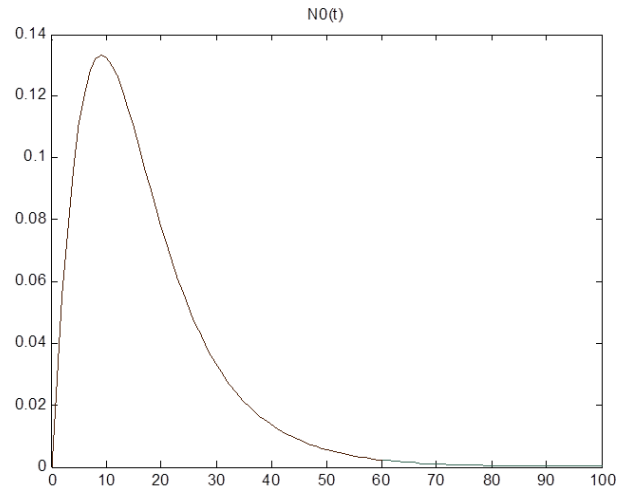

Figure 21. $N_{0}(t)$ for $\beta=0.45$.

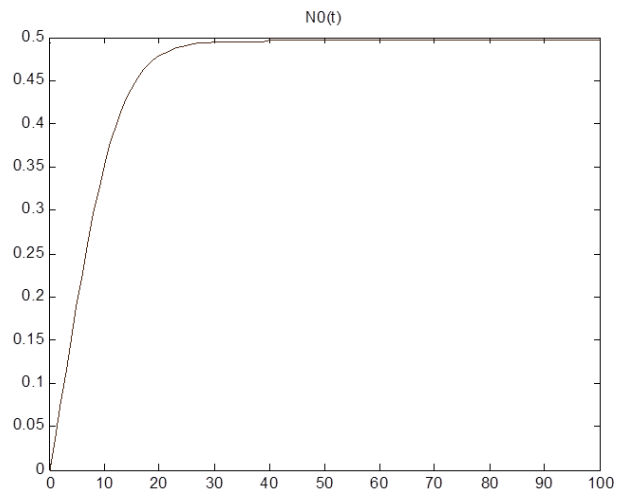

Figure 22. $N_{0}(t)$ for $\beta=0.5$.

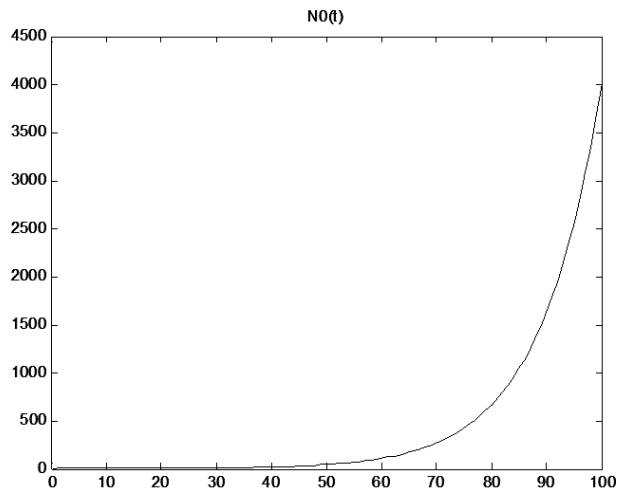

Figure 23. $N_{0}(t)$ for $\beta=0.55$. 
COMPARISON BETWEEN TWO APPROACHES TO MODELING MICROSATELLITE DNA REPEATS: INFINITE DIMENSIONAL MODEL AND ITS $N$-DIMENSIONAL APPROXIMATION

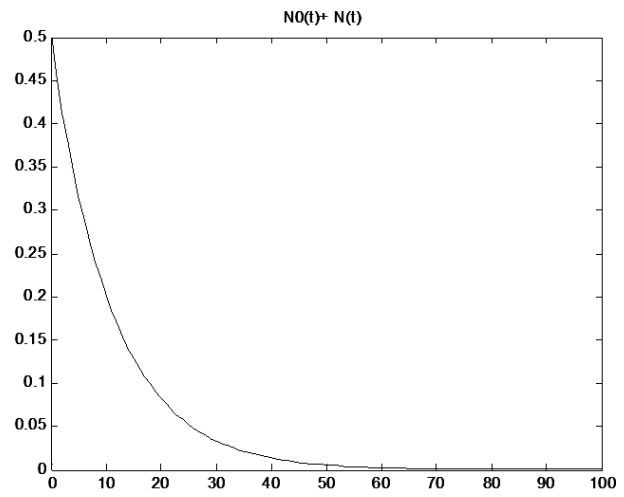

Figure 24. $\sum_{i=0}^{5} N_{i}(t)$ for $\beta=0.45$.

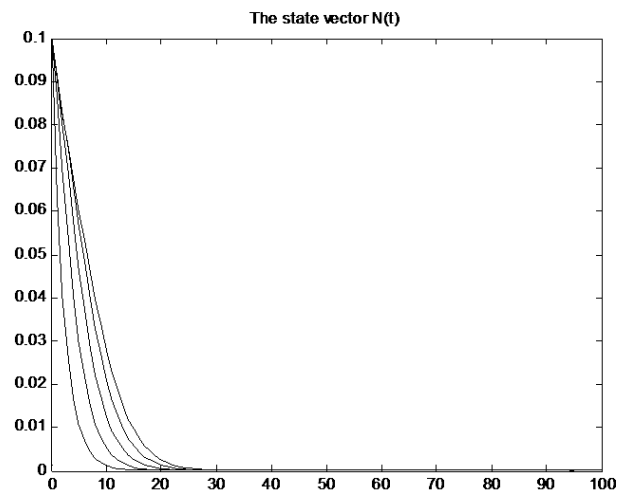

Figure 25. The state vector $N(t)$ for $\beta=0.5$.

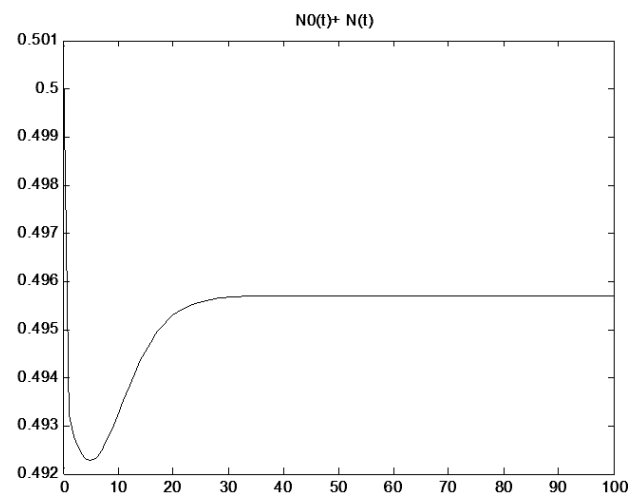

Figure 26. $\sum_{i=0}^{5} N_{i}(t)$ for $\beta=0.5$. 


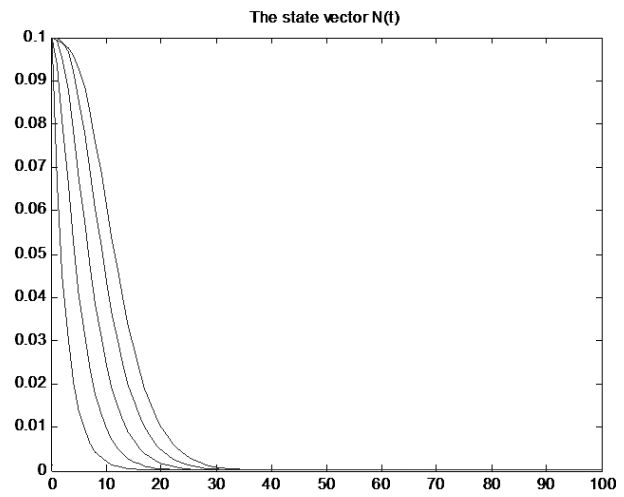

Figure 27. The state vector $N(t)$ for $\beta=0.5$.

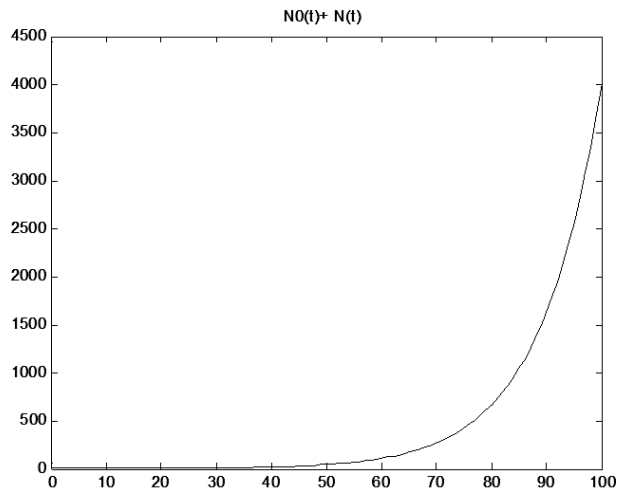

Figure 28. $\sum_{i=0}^{5} N_{i}(t)$ for $\beta=0.55$.

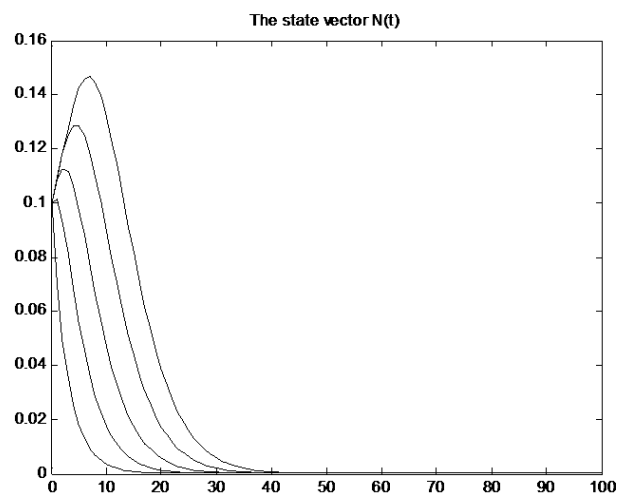

Figure 29. The state vector $N(t)$ for $\beta=0.55$. 
COMPARISON BETWEEN TWO APPROACHES TO MODELING MICROSATELLITE DNA REPEATS: INFINITE DIMENSIONAL MODEL AND ITS $N$-DIMENSIONAL APPROXIMATION

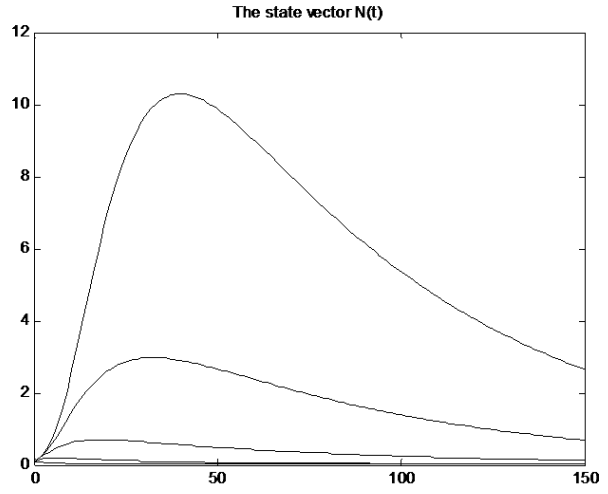

Figure 30. The state vector $N(t)$ for $\beta=0.75$.

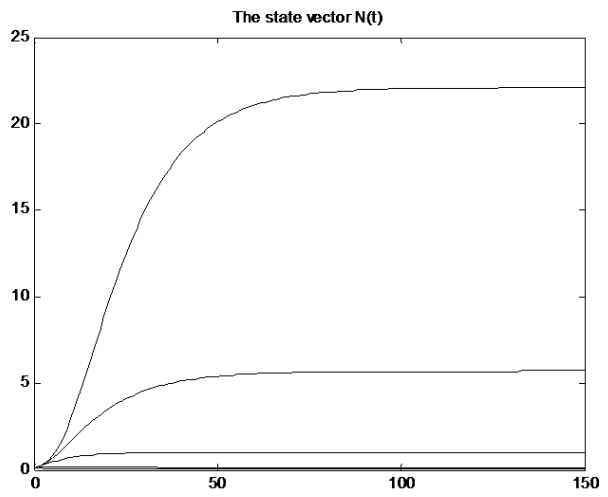

Figure 31. The state vector $N(t)$ for $\beta=\beta_{1}$.

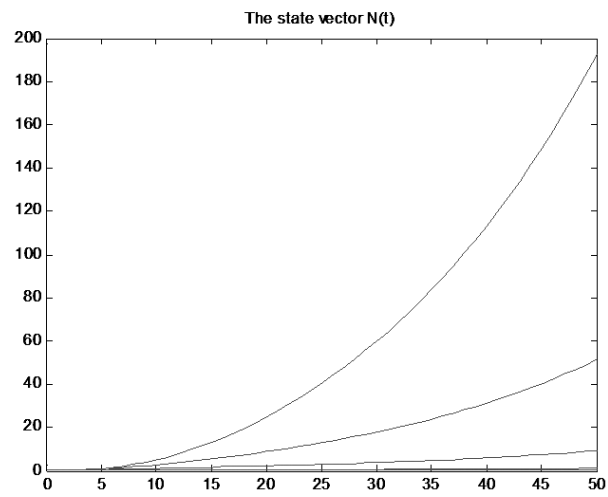

Figure 32. The state vector $N(t)$ for $\beta=0.8$. 


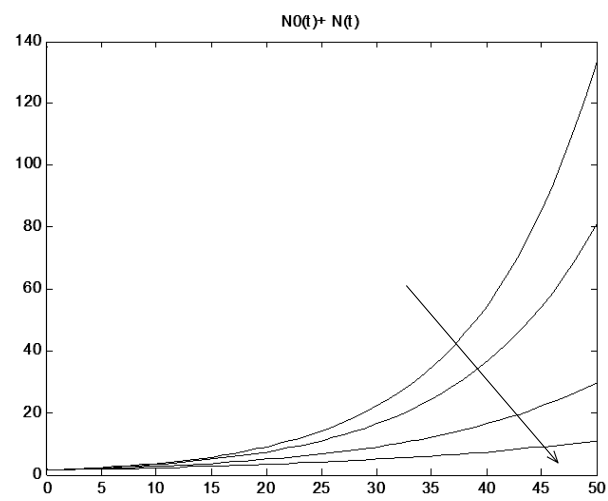

Figure 33. $\sum_{i=0}^{5} N_{i}(t)$ for $\theta=0.9,0.8,0.60 .4$.

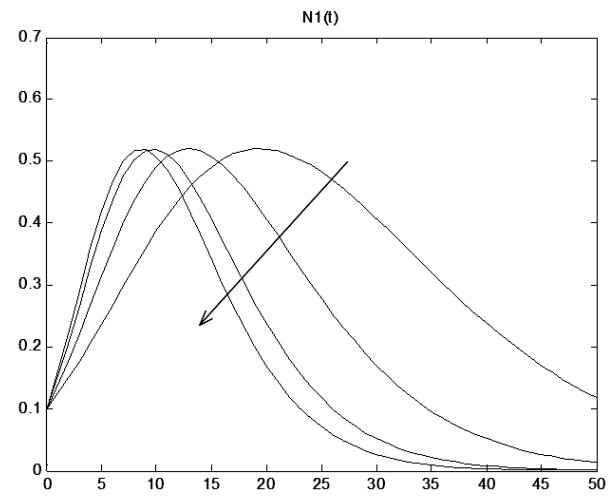

Figure 34. $N_{1}(t)$ for $\theta=0.9,0.8,0.60 .4$.

When $\beta$ grows and tends to the value of $\beta_{1}$ we observe slower convergence to 0 (Fig. 30). For $\beta=\beta_{1}$ the solution settles on certain value (Fig. 31). In Fig. 32 we have $\beta>\beta_{1}$ and the solution becomes unstable.

Now we will check how the changes of $\theta$ effect the solution (Figs 33 and 34). We assume the following parameter values: $n=5, \beta=0.55, v=0.45, \eta=0.01$, vector of initial conditions $N(0)=\left[\begin{array}{lllll}0.1 & 0.2 & 0.3 & 0.4 & 0.5\end{array}\right], N_{0}(0)=0$ and $\theta=0.9,0.8,0.6,0.4$. Parameter $\theta$ is inversely proportional to particle lifetime, thus the smaller $\theta$ the later cell division takes place. Parameter $\theta$ neither effects the quantity of particles nor changes the stability, but its decrease slows down the decay of the population.

We need now to evaluate the influence of $v$ (the probability of the progeny of $i$ type particle will become of type $i-1$ ), and $\eta$ (the probability that the progeny of $i$ type particle become of type $i+1)$. Both of these parameters have the same influence on the value of $\beta_{1}$ determined by (21). The level lines of $\beta_{1}$ in relation to $v$ and $\eta$ (for $n=5$ ) are 


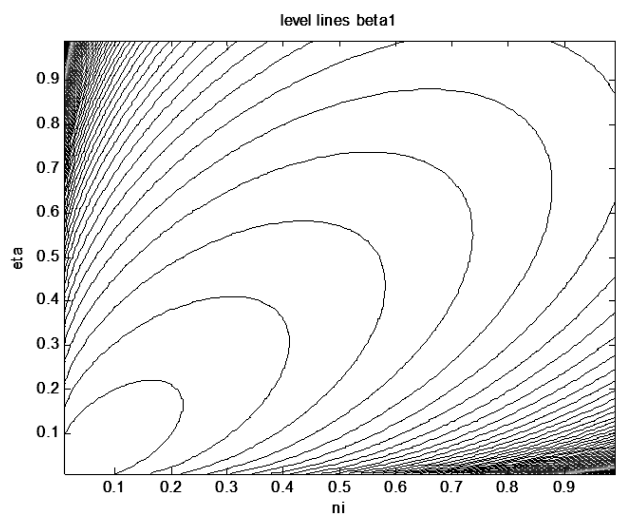

Figure 35. Level lines of $\beta_{1}(v, \eta)$.

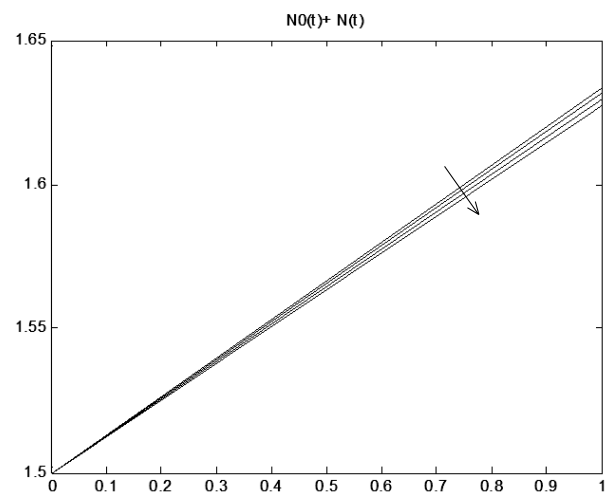

Figure 36. $\sum_{i=0}^{5} N_{i}(t)$ for $v=0.45,0.35,0.25,0.15$.

presented in the Fig. 35. Previously undertaken assumptions (3) limit the possible values of $v$ and $\eta$ to values from the area below the line described by the equation $v=\eta$.

Figures 36 and 37 represent the behavior of the solution for different values of $v$ $(0.45,0.35,0.25,0.15)$ and $\eta=0.01$ (we assume $\theta=0.9$, the other parameter are as above). Notice, that the increase of $v$ results in growth of $\beta_{1}$ and its decrease leads to the loss of stability of the solution.

Now we can see (Figs 38 and 39) the dependence of the solution on different values of $\eta(0.01,0.1,0.2,0.3)$ and $v=0.45$. We assume: $n=5, \theta=0.9, \beta=0.55$, vector of initial conditions $N(0)=\left[\begin{array}{lllll}0.1 & 0.2 & 0.3 & 0.4 & 0.5\end{array}\right], N_{0}(0)=0$. The increase of $\eta$ causes the lower value of $\beta_{1}$ and slower decay of particles quantity. 


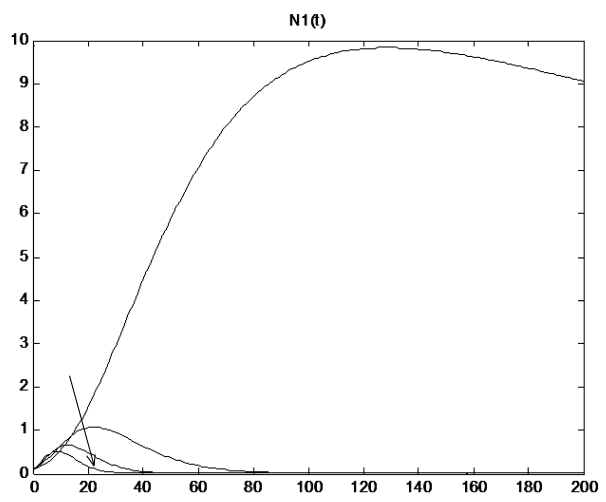

Figure 37. $N_{1}(t)$ for $v=0.45,0.35,0.25,0.15$.

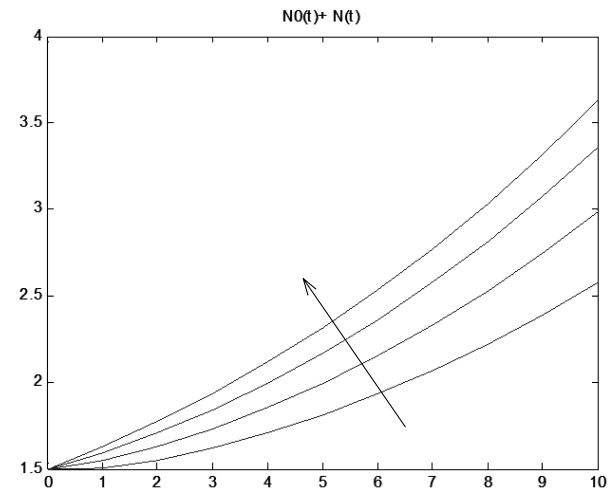

Figure 38. $\sum_{i=0}^{5} N_{i}(t)$ for $\eta=0.0 .01,0.1,0.2,0.3$.

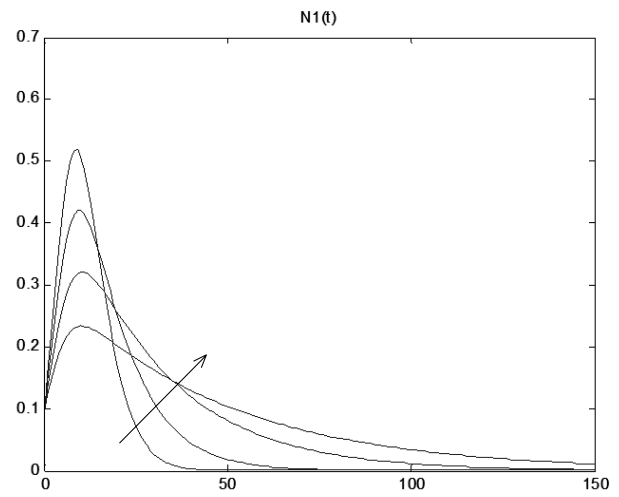

Figure 39. $N_{1}(t)$ for $\eta=0.0 .01,0.1,0.2,0.3$. 


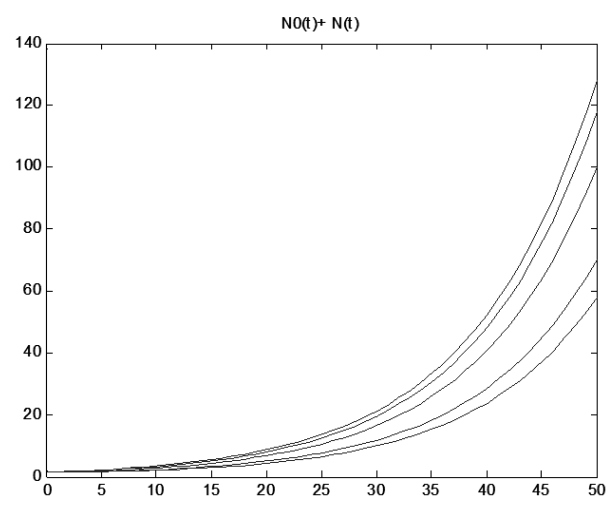

Figure $40 . \sum_{i=0}^{5} N_{i}(t)$.

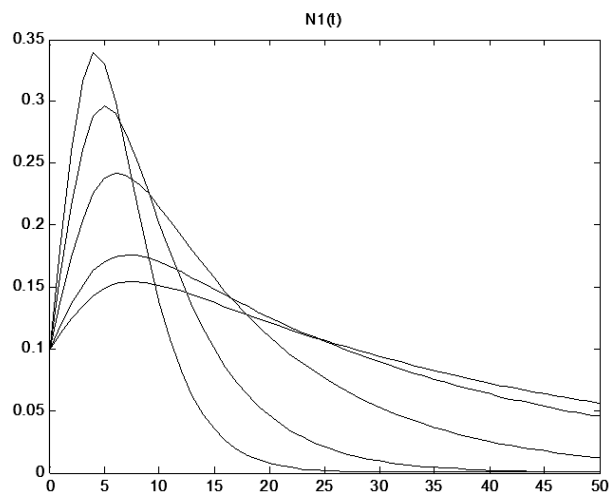

Figure 41. $N_{1}(t)$.

We can see in figures 40 and 41 that when the difference between the $v$ and $\eta$ values increases then the solution decay becomes faster (we assume: $n=5, \theta=0.9, \beta=0.55$, vector of initial conditions $N(0)=\left[\begin{array}{lllll}0.1 & 0.2 & 0.3 & 0.4 & 0.5\end{array}\right], N_{0}(0)=0, v=0.8,0.7,0.6$, $0.5,0.45, \eta=0.1,0.2,0.3,0.4,0.42)$.

\section{Concluding remarks}

In this paper we have studied the properties of two models describing evolution of microsatellite DNA repeats. The infinite dimensional model in the form of infinite dimensional system of linear differential equations was analyzed by using techniques of inverse Laplace transforms for non-rational functions and asymptotic expansions of 
integrals and functions. The resulting formulas enable us to make numerical calculations which allow to determine the influence of system parameters on the trajectory of the solution which is the same in both considered cases. The significant difference between both models is that the unstable trajectories of the $n$-dimensional solution do not show the loss of the value in the short time horizon. The growth of particles quantity takes place immediately. However in the case of infinite system the analytical form of the accurate solution makes it difficult to evaluate the parameters influence, it can be defined only by numerical studies. The results correspond to conclusions coming from the analysis of the convergence condition $\sqrt{d}-\sqrt{b}>\sqrt{\lambda}$. The increase of the values of $b$ and $\lambda$ leads to the loss of solution convergence, while the increase of value of $d$ accelerates the decay of the solution. As to $n$-dimensional approximation the influence of $\beta$ comes directly from the stability condition (21). The results of numerical studies confirm the character of $\theta$ influence. Parameter $\theta$ does not changes the $\beta_{1}$ value (21) but influences the eigenvalues of $\left[\lambda I+A_{n}\right]$ defined by (12), therefore the decrease of $\theta$ slows down the solution decay. It is important to notice, that the effect of changes of $v$ and $\eta$ values in case of $n$-dimensional approximation is corresponding to the influence of parameters $d$ and $b$ of infinite system only in the range of $v$ and $\eta$ values satisfying condition $v>$ $\eta$. For $v<\eta$ the finite approximation remains asymptotically stable and the $v$ and $\eta$ influence is reversed (see Fig. 35). We need to mention that the numerical simulations of infinite model does not give the appropriate results for long range horizon because the trajectory of the solution below satisfied convergence conditions tends to "escape" to infinity. The decrease of integration step improves the results but slows down the calculation time. The numerical studies of $n$-dimensional approximation have also some restrictions concerning the size of the state vector. For $n \geqslant 16$ the matrix $P e^{\Lambda t} P^{-1}$ where $P=\left[w_{1}, \ldots, w_{n}\right], w(i)$ are eigenvectors of $\left[\lambda I+A_{n}\right]$ and $\Lambda=\left[\begin{array}{ccc}\lambda_{1} & 0 & 0 \\ 0 & \ddots & 0 \\ 0 & 0 & \lambda_{n}\end{array}\right]$ where $\lambda_{i}$ is eigenvalue of $\left[\lambda I+A_{n}\right]$ becomes close to singular and results are inaccurate.

\section{References}

[1] T.A. Brown: Genomes 3. Wydawnictwo Naukowe PWN, Warszawa, 2009, (in Polish).

[2] R. KORDEK and J. BARTKOWIAK: Microsatellite instability in human carcinomas. Onkol. Pol, 22, (1999), 109-112 (in Polish).

[3] M. Kimmel, A. ŇwierniaK and A. Polañski: Infinite-dimensional model of evolution of drug resistance of cancer cells. J. of Mathematical Systems, Estimation and Control, 8(1), (1998). 
COMPARISON BETWEEN TWO APPROACHES TO MODELING MICROSATELLITE DNA REPEATS: INFINITE DIMENSIONAL MODEL AND ITS N-DIMENSIONAL APPROXIMATION

[4] W. Mitkowski: Dynamic properties of chain systems with applications to biological models. Archives of Control Sciences, 9(1-2), (1999), 123-131.

[5] A. Bobrowski, M. Kimmel: Dynamics of the life history of a DNA-repeat sequence, Archives of Control Sciences Volume 9(XLV), 1999, No. 1-2, pages 57-67,

[6] R.M. Sibly, J.C. Whittaker and M. Talbot: A maximum-likelihood approach to fitting equilibrium models of microsatellite evolution. Mol. Biol. Evol., 18(3), (2001), 413-417.

[7] M. Kimmel and D.N. STIVERS: Time-continuous branching walk models of unstable gene amplification. Bull. Math. Biol., 56 (1994), 337-357.

[8] J. OsIOwSKI: Principles of operator calculus. WNT, Warszawa, 1981, (in Polish).

[9] B. WiNDLE and G.M. WAHL: Molecular dissection of mammalian gene amplification: New mechanistic insights revealed by analysis of very early events. Mutat. Res., 276 (1992), 199-224.

[10] W.P. ILIN and YU.I. KuZNYETSOW: Tridiagonal matrices and their applications. Nauka, Moskwa, 1985. 